ARTICLE

\title{
Establishing the carrier scattering phase diagram for ZrNiSn-based half-Heusler thermoelectric materials
}

\author{
Qingyong Ren (1) ${ }^{1}$, Chenguang Fu (i) ${ }^{2 凶}$, Qinyi Qiu ${ }^{3}$, Shengnan Dai ${ }^{4}$, Zheyuan Liu', Takatsugu Masuda (D) ${ }^{5}$, \\ Shinichiro Asai ${ }^{5}$, Masato Hagihala $\mathbb{1}^{6}{ }^{6}$, Sanghyun Lee ${ }^{6}$, Shuki Torri ${ }^{6}$, Takashi Kamiyama (0) 6,7, Lunhua He ${ }^{8,9,10}$, \\ Xin Tong (1) 10,11, Claudia Felser (1) ${ }^{2}$, David J. Singh (1) ${ }^{12}$, Tiejun Zhu (1) ${ }^{3}$, Jiong Yang (D) ${ }^{4 凶} \&$ Jie Ma (1) ${ }^{1,13 凶}$
}

Chemical doping is one of the most important strategies for tuning electrical properties of semiconductors, particularly thermoelectric materials. Generally, the main role of chemical doping lies in optimizing the carrier concentration, but there can potentially be other important effects. Here, we show that chemical doping plays multiple roles for both electron and phonon transport properties in half-Heusler thermoelectric materials. With ZrNiSn-based half-Heusler materials as an example, we use high-quality single and polycrystalline crystals, various probes, including electrical transport measurements, inelastic neutron scattering measurement, and first-principles calculations, to investigate the underlying electron-phonon interaction. We find that chemical doping brings strong screening effects to ionized impurities, grain boundary, and polar optical phonon scattering, but has negligible influence on lattice thermal conductivity. Furthermore, it is possible to establish a carrier scattering phase diagram, which can be used to select reasonable strategies for optimization of the thermoelectric performance.

\footnotetext{
${ }^{1}$ Key Laboratory of Artificial Structures and Quantum Control, School of Physics and Astronomy, Shanghai Jiao Tong University, 800 Dongchuan Road, 200240 Shanghai, China. ${ }^{2}$ Max Planck Institute for Chemical Physics of Solids, Nöthnitzer Straße 40, Dresden 01187, Germany. ${ }^{3}$ State Key Laboratory of Silicon Materials, School of Materials Science and Engineering, Zhejiang University, 310027 Hangzhou, China. ${ }^{4}$ Materials Genome Institute, Shanghai University, 99 Shangda Road, 200444 Shanghai, China. ${ }^{5}$ Neutron Science Laboratory, Institute for Solid State Physics, University of Tokyo, Kashiwanoha, Kashiwa 277-8581, Japan. ${ }^{6}$ Institute of Materials Structure Science, High Energy Accelerator Research Organization (KEK), Tokai, Ibaraki 319-1106, Japan. ${ }^{7}$ Department of Materials Structure Science, Sokendai (The Graduate University for Advanced Studies), Tokai, Ibaraki 319-1106, Japan. ${ }^{8}$ Beijing National Laboratory for Condensed Matter Physics, Institute of Physics, Chinese Academy of Sciences, 100190 Beijing, China. ${ }^{9}$ Songshan Lake Materials Laboratory, 523808 Dongguan, Guangdong, China. ${ }^{10}$ Spallation Neutron Source Science Center, 523803 Dongguan, China. ${ }^{11}$ Institute of High Energy Physics, Chinese Academy of Sciences, 100049 Beijing, China. ${ }^{12}$ Department of Chemistry and Department of Physics and Astronomy, University of Missouri-Columbia, Columbia, MO 65211, USA. ${ }^{13}$ Shenyang National Laboratory for Materials Science, Institute of Metal Research, Chinese Academy of Sciences, 110016 Shenyang, China. ${ }^{凶}$ email: Chenguang.Fu@cpfs.mpg.de; jiongy@t.shu.edu.cn; jma3@sjtu.edu.cn
} 
T hermoelectric (TE) materials enable direct conversion between heat and electricity. They can be used to generate electricity based on the Seebeck effect when a thermal gradient exists or to transfer heat against temperature gradient based on the Peltier effect when an electric current is applied ${ }^{1}$. Many thermoelectric materials are being explored for power generation applications, such as half-Heusler ${ }^{2}, \mathrm{PbTe}^{3-5}$, silicides ${ }^{6}$, $\mathrm{CoSb}_{3}{ }^{7}$, and $\mathrm{Mg}_{3} \mathrm{Sb}_{2}{ }^{8}$. The energy conversion efficiency for TE materials depends heavily on the TE material performance. Practical applications require further improvement on the performance of TE materials. This is quantified by the thermoelectric figure of merit, $z T=\alpha^{2} \sigma T /\left(\kappa_{\text {ele }}+\kappa_{\text {lat }}\right)$, where $\alpha, \sigma, T$, $\kappa_{\text {ele }}$, and $\kappa_{\text {lat }}$ are the Seebeck coefficient, the electrical conductivity, the absolute temperature, the electronic thermal conductivity, and the lattice thermal conductivity, respectively ${ }^{9}$. Good TE materials generally have features as Phonon Glass Electron Crystal, which means good electrical transport properties, characteristic of crystalline semiconductors, and a low thermal conductivity, characteristic of glasses ${ }^{10}$.

The optimization of TE performance is complex, requiring manipulation and optimization of both the electrical and thermal transport ${ }^{11}$. This includes controlling the underlying scattering mechanisms, e.g. alloy scattering ${ }^{12}$, ionized impurity scattering ${ }^{13}$, carrier-carrier scattering ${ }^{14}$, acoustic and optical phonon scatterings ${ }^{15}$ for the charge carriers, and phonon anharmonicity ${ }^{16-18}$, atomic rattling ${ }^{19}$, nanostructuring ${ }^{20}$, and glass-like or superionic behavior $^{21-23}$ for the phonons. Due to the diverse temperature dependences of the scattering mechanisms, electrical and thermal conductivities, in general, exhibit different temperature dependences. For instance, the carrier mobility follows $\mu_{\mathrm{ac}} \propto T^{-3 / 2}$ for acoustic phonon dominated scattering, $\mu_{\mathrm{pe}} \propto T^{-1 / 2}$ for piezoelectric scattering, or $\mu_{\mathrm{i}} \propto T^{3 / 2}$ for ionized impurity scattering ${ }^{24}$, while the lattice thermal conductivity follows $\kappa_{U} \propto T^{-1}$ for Umklapp scattering or $\kappa_{\mathrm{al}} \propto T^{-1 / 2}$ for alloy scattering ${ }^{25,26}$.

On the other hand, the scattering rates for the carrier scattering mechanisms also show strong carrier concentration dependences ( $n$ for electron concentration, $p$ for hole concentration), and may be more complex than the standard parabolic band expressions noted above. As an example, changes in carrier concentration could alter the carrier energy or carrier density of states around the Fermi level, and hence influence the acoustic phonon scattering and the mobility ${ }^{27}$. As demonstrated in Fig. 1a, the carrier transport in most TE semiconductors, except the lead chalcogenides, are dominated by the phonon scattering, and the mobility shows almost monotonous decrease with the carrier concentration, $n$ or $p^{15,28-31}$. The temperature and carrier concentration dependences of the scattering provide possibilities for the manipulation of the transport properties. Therefore, mapping of a carrier scattering phase diagram in the space of temperature and carrier concentration would provide a useful guidance for optimization or design of TE materials, such as the study of BXGa $(\mathrm{X}=\mathrm{Be}, \mathrm{Mg}$, and $\mathrm{Ga})$ compounds $^{32}$.

Half-Heusler (HH) TE compounds, which are used for promising power generation applications, have attracted extensive attentions in recent years ${ }^{33,34}$. Since the study of $\mathrm{ZrNiSn}$ compounds ${ }^{35-37}$, large $z T$ values above unity can be obtained in both $n$-type and $p$-type $\mathrm{HH}$ alloys 38,39 . The carrier transport mechanism underlying their high TE performance has been a focus of study. Current studies of the carrier transport mechanisms in half-Heusler compounds mainly focus on acoustic phonons, alloy, and grain boundary scatterings. These exhibit distinct temperature dependences ${ }^{12,40-42}$. On the other hand, the carrier concentration dependent carrier mobility, $\mu(n / p)$, of $\mathrm{HH}$ compounds has been rarely studied. As summarized in Fig. 1b, $\mu(n / p)$ of both $n$-type and $p$-type $\mathrm{HH}$ compounds exhibits a nonmonotonous behavior and the peak $\mu(n / p)$ usually occurs near to
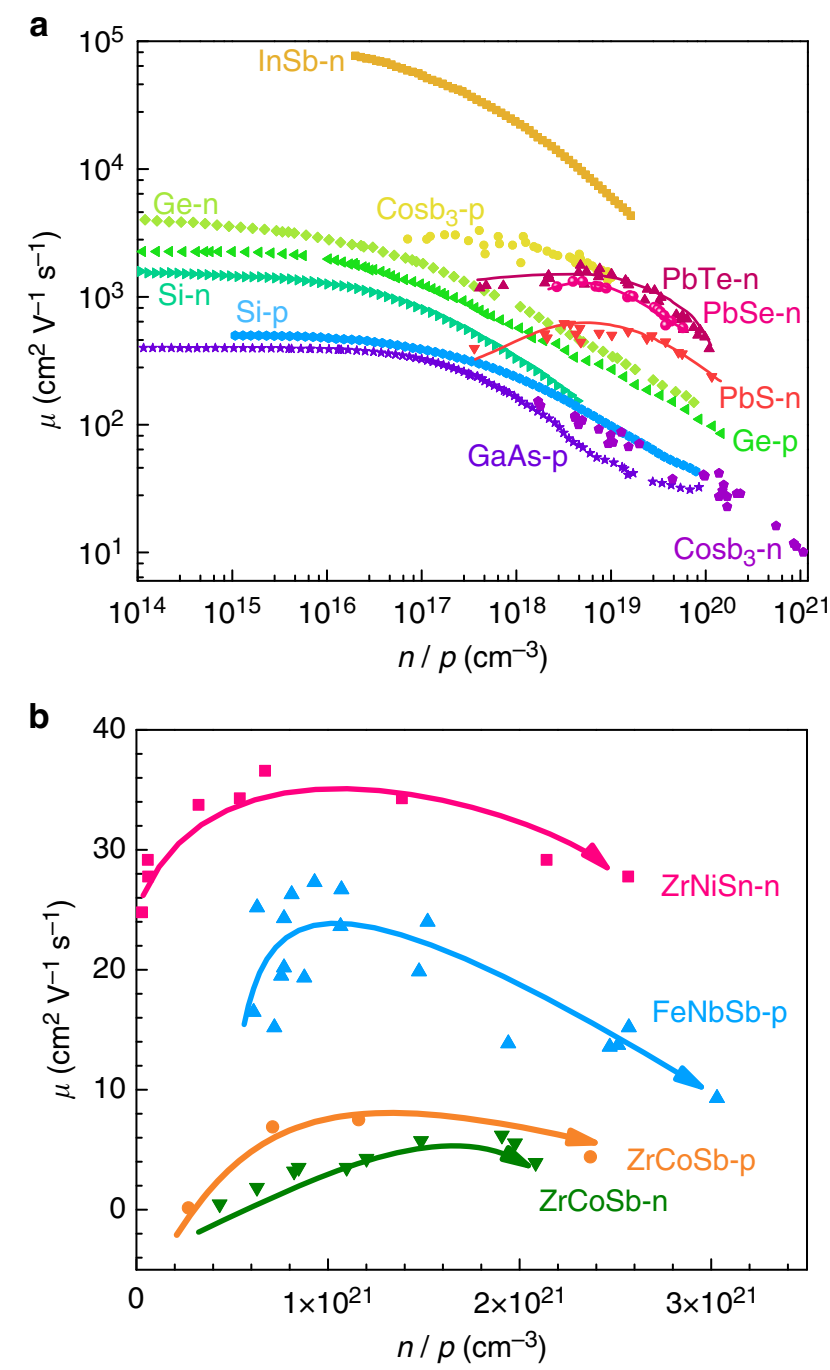

Fig. 1 Room temperature carrier mobility versus carrier concentration n/p. a Some selected TE semiconductors ${ }^{15}, 28-31$, and $\mathbf{b}$ several half-Heusler TE compounds $40-43$.

the optimal $n / p$ of the system, where the $z T$ peaks ${ }^{40-43}$. This indicates that the carrier scattering mechanisms in $\mathrm{HH}$ compounds have strong dependences on the carrier concentration. The decrease of $\mu(n / p)$ at high $n / p$ region is generally thought to be a result of the acoustic phonon scattering, as exhibited in Fig. 1a. However, the increase of $\mu(n / p)$ in the low $n / p$ region suggests some other important scattering mechanisms, which are not well understood and motivate further study.

Here, we use high-quality single and polycrystalline samples, and various probes, including electrical and thermal transport measurements, neutron powder diffraction (NPD) and inelastic neutron scattering (INS) measurements, and first-principles calculations, to identify the electron and phonon transport behavior of Sb-doped $\mathrm{ZrNiSn}$, a representative HH TE system. We reveal important scattering beyond the acoustic phonon scattering. Specifically, ionized impurity, grain boundary, and polar optical phonon scatterings are shown to contribute significantly in controlling the electrical transport. Moreover, the effect of electron-phonon interactions on lattice thermal conductivity is comprehensively analyzed. Based on these results and analysis, a carrier scattering phase diagram is established. This could serve as a guide in further improvement of the TE performance in $\mathrm{HH}$ compounds. 

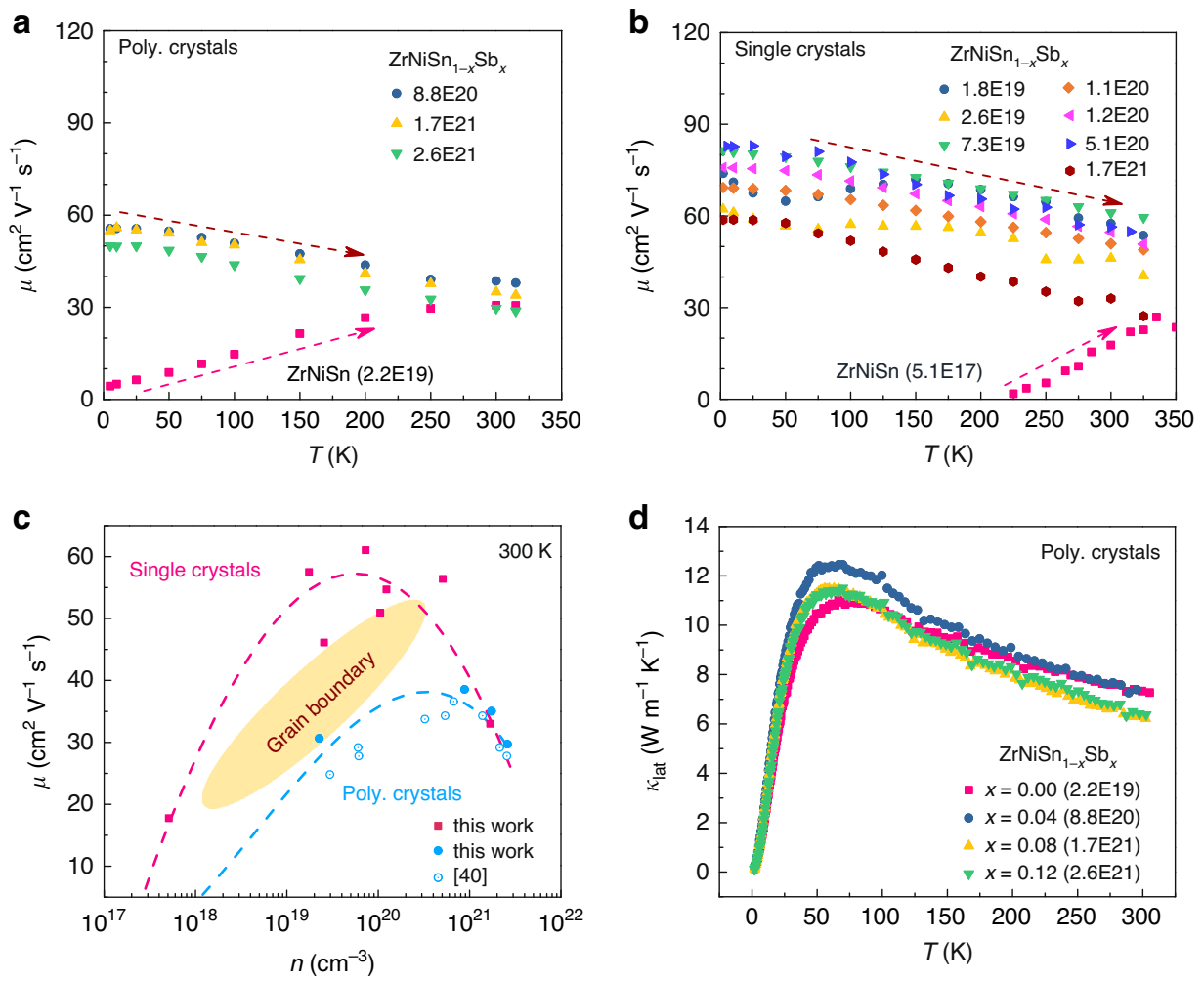

Fig. 2 Electrical and thermal transport properties for the parent and $\mathbf{S b}$-doped $\mathbf{Z r N i S n} \mathbf{n}_{\mathbf{1}-\mathbf{x}} \mathbf{S b}_{\mathbf{x}}$. Carrier concentration dependent mobility for a polycrystalline samples and $\mathbf{b}$ single crystals. c Carrier mobility versus carrier concentration for $\mathrm{ZrNiSn}_{1-x} \mathrm{Sb}_{x}$ at $300 \mathrm{~K}$. The dash lines in the figures are guides of the eye. $\mathbf{d}$ Lattice thermal conductivity, $\kappa_{\text {lat, }}$ of the polycrystalline samples over the temperature range of $2 \mathrm{~K}$ to $300 \mathrm{~K}$.

\section{Results}

Electrical and thermal transport properties. High-quality $\mathrm{ZrNiSn}_{1-x} \mathrm{Sb}_{x}$ polycrystalline samples were made by levitation melting and spark plasma sintering (see Methods for details). All the polycrystalline samples contain $5-7 \%$ more interstitial $\mathrm{Ni}$ on the $4 d(3 / 4,3 / 4,3 / 4)$ site (Supplementary Fig. 1), being consistent with the previous electron probe microanalysis ${ }^{40}$. Temperature dependences of the mobility in these polycrystalline samples were studied over the temperature range of $5 \mathrm{~K}$ to $315 \mathrm{~K}$ (variation of $n_{\mathrm{H}}$ with $T$ and Sb content are shown in Supplementary Fig. 3). $\mu(T)$ of Sb-doped samples monotonically decreases with temperature (Fig. 2a), since the acoustic phonon and alloy scatterings contribute importantly to the electron scattering. Meanwhile, the undoped ZrNiSn parent sample exhibits a positive dependence of $\mu(T)$ on temperature, opposite to the doped samples. Similar to $n$-type $\mathrm{Mg}_{3}(\mathrm{Sb}, \mathrm{Bi})_{2}$ system, this kind of increasing trend of $\mu(T)$ raises the issue of the carrier scattering mechanism in underdoped TE materials with low carrier concentration or with small grain size ${ }^{44,45}$, i.e., does the increased $\mu(T)$ below the crossover temperature stems from the grain boundary scattering or ionized impurity scattering ${ }^{44,45}$ ?

One direct way to answer this question would be a comparative study of the temperature-dependent electrical conductivity of both polycrystalline and single-crystalline samples, thereby addressing the issue of grain boundary scattering. High-quality single crystalline $\mathrm{ZrNiSn}_{1-x} \mathrm{Sb}_{x}$ samples were then grown by a $\mathrm{Sn}$ flux method ${ }^{46}$ (details are given in Methods). The corresponding $\mu(T)$ is presented in Fig. 2b. The $\mu(T)$ of the undoped ZrNiSn single crystal, which by nature does not have grain boundaries, still shows an increasing trend with $T$. This indicates that ionized impurities might dominate the electron transport. The $\mu(T)$ for the $\mathrm{Sb}$-doped $\mathrm{ZrNiSn}_{1-x} \mathrm{Sb}_{x}$ crystals exhibits a decreasing trend with $T$, similar to the doped polycrystalline samples (Fig. 2a). The carrier concentration dependent $\mu(n)$ at $300 \mathrm{~K}$ of both polycrystalline and single-crystalline samples is displayed in Fig. 2c. The $\mu(n)$ of single crystals is larger than that of polycrystalline samples at low $n$ region $\left(<10^{21} \mathrm{~cm}^{-3}\right)$ while they converge at the high $n$ heavily doped region $\left(>10^{21} \mathrm{~cm}^{-3}\right)$, indicating that grain boundaries also play a significant role in scattering electrons of lightly doped polycrystalline samples. Additionally, the nonmonotonous dependence of $\mu(n)$, as observed in the representative polycrystalline $\mathrm{HH}$ systems (Fig. 1b), is also shown in the single crystalline $\mathrm{ZrNiSn}_{1-x} \mathrm{Sb}_{x}$ samples.

We now turn to the thermal transport. The lattice thermal conductivity $\kappa_{\text {lat }}$ of polycrystalline $\mathrm{ZrNiSn}_{1-x} \mathrm{Sb}_{x}$ samples over the temperature range of $2 \mathrm{~K}$ to $300 \mathrm{~K}$ is shown in Fig. $2 \mathrm{~d}$ (temperature dependences of the electronic resistivity $\rho$, Lorenz number $L$, electronic thermal conductivity $\kappa_{\text {ele }}$, and total thermal conductivity $\kappa_{\text {tot }}$ are provided in Supplementary Fig. 4). It is surprisingly found that over the whole studied temperature range $\mathrm{Sb}$ doping does not show a significant effect on the $\kappa_{\text {lat }}$ of $\mathrm{ZrNiSn}$. This is true even for the extreme case where $12 \%$ of $\mathrm{Sn}$ was replaced by aliovalent $\mathrm{Sb}$. The atomic mass and radius of $\mathrm{Sb}$ are close to those of Sn. Nonetheless, the fact that such a high content of aliovalent doping does not obviously affect the phonon propagation is unexpected, since in many solid materials even partial isotopic substitution can lead to a significant changes in the $\kappa_{\mathrm{lat}}{ }^{47,48}$. In the very similar case of Sn-doped $\mathrm{ZrCoSb}_{1-x} \mathrm{Sn}_{x}$ $\mathrm{HH}$ compounds, replacement of $10 \%$ of the Sb by Sn results in a large reduction of $\sim 45 \%$ in the $\kappa_{\text {lat }}$ near room temperature ${ }^{43}$. Similarly, in $p$-type NbFeSb-based compounds, aliovalent doping also significantly suppresses the lattice thermal conductivity $^{33,49}$. Therefore, the present result together with the nonmonotonous dependence of $\mu(n)$ motivate further exploration of the underlying electron and phonon transport mechanisms in the $\mathrm{ZrNiSn}_{1-x} \mathrm{Sb}_{x}$ system. 

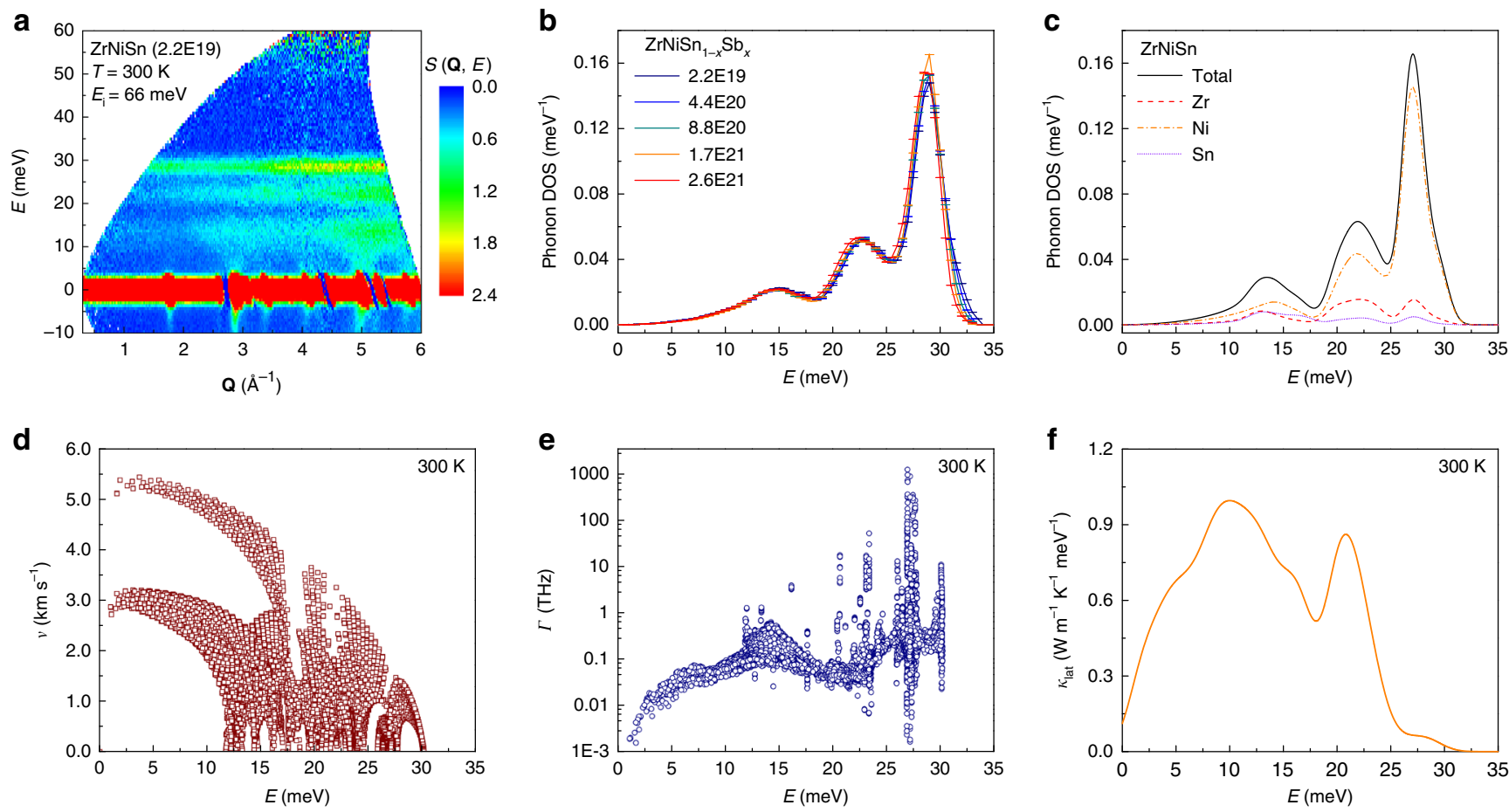

Fig. 3 Phonon DOSs and calculated lattice thermal conductivity. a Experimental dynamical structure factor, $S(\mathbf{Q}, E)$, measured with inelastic neutron scattering for the $\mathrm{ZrNiSn}$ sample with $E_{\mathrm{i}}=66 \mathrm{meV}$. b Neutron-weighted phonon DOS of the $\mathrm{ZrNiSn}_{1-x} \mathrm{Sb}_{x}$ compounds with different carrier concentration $n\left(\mathrm{~cm}^{-3}\right)$. c Total and partial neutron-weighted phonon DOS for ZrNiSn obtained from first-principles calculations. $\mathbf{d}$ Calculated group velocities, $\nu$, and e scattering rate, $\Gamma$, for ZrNiSn. $\mathbf{f}$ calculated energy-dependent lattice thermal conductivity for ZrNiSn at $300 \mathrm{~K}$.

Phonon density of states. To understand the effects of electron doping on the electron and phonon transport properties in ZrNiSn-based $\mathrm{HH}$ compounds, it is necessary to consider electron-phonon interactions. We begin with the phonons. Phonon density of states (DOSs) measurements for $\mathrm{ZrNiSn}_{1-x} \mathrm{Sb}_{x}$ with different electron concentrations were carried out on the High-Resolution Chopper Spectrometer, BL12 HRC, at MFL of JPARC. The momentum and energy dependence of powderaveraged dynamical structure factor, $S(\mathbf{Q}, E)$, of $\mathrm{ZrNiSn}$ at $300 \mathrm{~K}$, as an example, are shown in Fig. 3a. The phonon signal shows normal $\mathbf{Q}^{2}$ dependence. The neutron-weighted phonon DOSs were obtained by integration of the $S(\mathbf{Q}, E)$ over the range of momentum transfers $0.4 \leq \mathbf{Q} \leq 6.0 \AA^{-1}$. The carrier concentration dependence of neutron-weighted phonon DOSs of $\mathrm{ZrNiSn}_{1-x} \mathrm{Sb}_{x}$ is shown in Fig. 3b. Three phonon bands, $0-18.5 \mathrm{meV}, 18.5-$ $25.5 \mathrm{meV}$, and $25.5-33 \mathrm{meV}$, are observed, corresponding to one acoustic phonon band and two optical phonon bands, respectively. Due to the different ratios of neutron scattering crosssection over atomic mass, $\sigma / m$, of the different elements, the motions of $\mathrm{Ni}$ are overemphasized while those for $\mathrm{Zr}$ and $\mathrm{Sn} / \mathrm{Sb}$ are underestimated. The phonon DOS as obtained from firstprinciples calculations shown in Fig. $3 \mathrm{c}$ for $\mathrm{ZrNiSn}$ with proper neutron weighting is in good agreement with experimental data (see Supplementary Fig. 5 for calculated phonon DOSs without neutron weighting).

Electron-phonon interaction and lattice thermal conductivity. A careful comparison of the phonon DOSs in Fig. 3b shows that the optical phonon bands exhibit clear changes with $n$. A prominent tail is present above $30 \mathrm{meV}$ for the parent $\mathrm{ZrNiSn}$ sample, which gradually becomes smaller with increasing $n$. However, the calculated phonon transport properties of $\mathrm{ZrNiSn}$ demonstrate that the optical phonons above $\sim 25 \mathrm{meV}$ have much smaller group velocity (Fig. 3d), and suffer stronger scattering
(Fig. 3e). Hence, they have a quite small contribution to the average lattice thermal conductivity, $\kappa_{\text {lat }},(<\sim 1.5 \%$, Fig. $3 \mathrm{f})$, and the changes in the optical phonons cannot make a palpable influence on $\kappa_{\text {lat }}$. In addition, the acoustic phonon band in Fig. $3 \mathrm{~b}$ is insensitive to changes in $n$. This is one indication of relatively weak coupling between electrons and acoustic phonons. This weak coupling could be attributed to the symmetry-protected non-bonding orbitals-electron states or impotent acoustic phonons deformation potentials ${ }^{50}$. Since the acoustic phonons are the main carriers for thermal energy with large group velocity (Fig. 3d) and small scattering rate (Fig. 3e), it might be the overall weak interaction between the electrons and heat-carrying acoustic phonons and the negligible contribution of the optical phonons (above $\sim 25 \mathrm{meV}$ ) that make the $\kappa_{\text {lat }}$ does not show obvious changes in the polycrystalline $\mathrm{ZrNiSn}_{1-x} \mathrm{Sb}_{x}$ compounds with different carrier concentrations or Sb contents (Fig. 2d).

LO-TO splitting and polar optical phonon scattering. We turn to the observed non-monotonous behavior of $\mu(n)$ (Figs. $1 \mathrm{~b}$ and 2c). The $25.5-33 \mathrm{meV}$ optical phonon band of the ZrNiSn parent sample, which contains the prominent tail, can be decomposed into two peaks as shown in Fig. 4a. To understand the origin of the tail, first-principles calculations of phonon dispersion are performed. As shown in Fig. 4b, the degeneracy of longitudinal optical (LO) phonon and transverse optical (TO) phonon at $\sim 28 \mathrm{meV}$ is lifted around the Brillouin zone center, corresponding to the LO-TO splitting. The peaks centered at $\sim 28.9 \mathrm{meV}$ in Fig. 4a corresponds to the TO branches, while the $\sim 31.9 \mathrm{meV}$ one represents the LO branch.

The LO-TO frequency (energy) difference is due to the longrange electric field in an insulator ${ }^{51}$. This polarization field is produced by the LO phonon vibration, which in turn can raise the frequency of LO branch (see methods for details). In addition, this polarization field also imposes a strong disturbance on the 

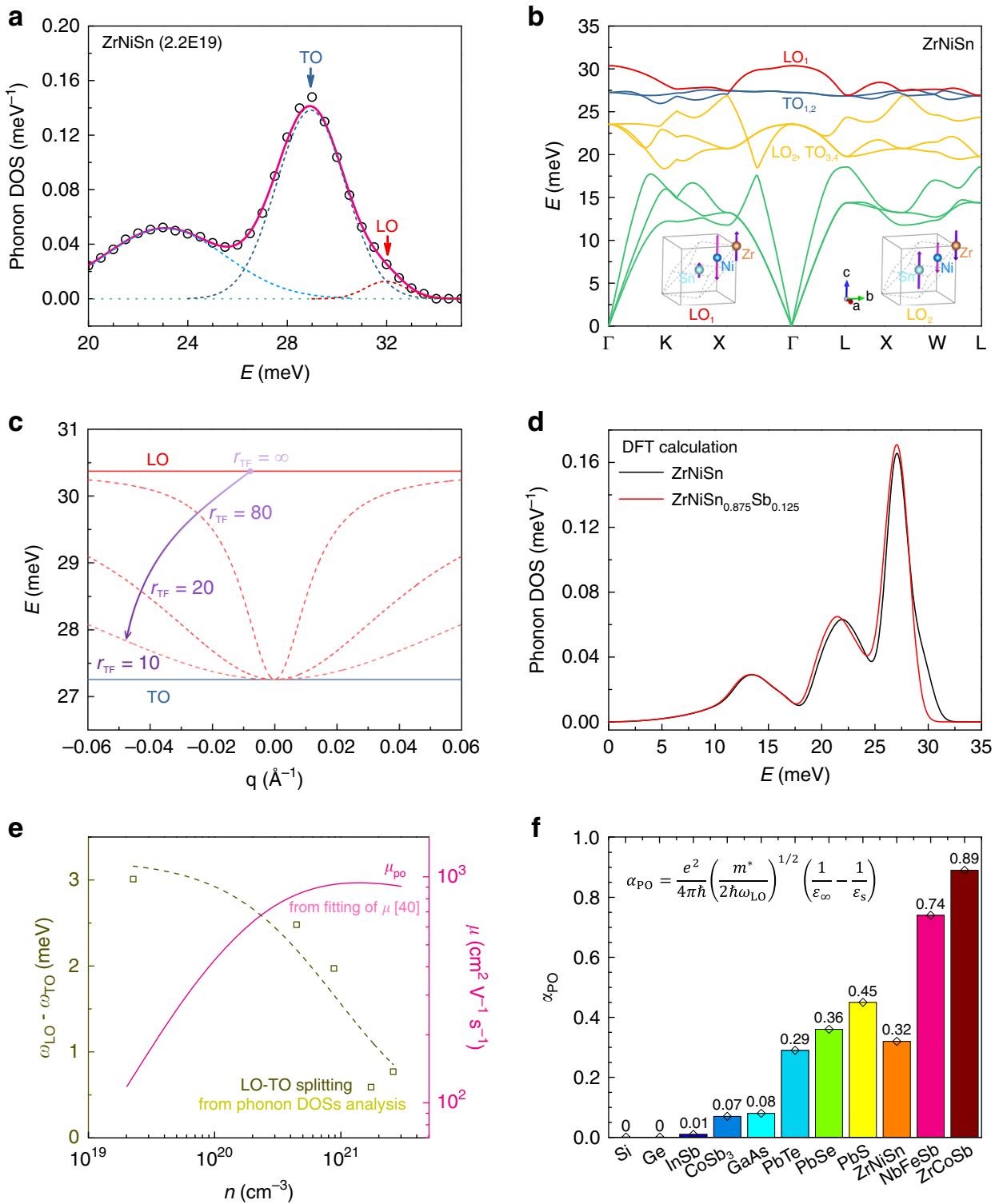

Fig. 4 Screening of the LO-TO splitting and polar optical scattering. a Decomposition of the optical phonon bands shows the LO-TO splitting. b Calculated phonon dispersion in ZrNiSn parent sample. The insets illustrate the phonon vibration modes for the two longitudinal optical phonons (a propagation vector along $c$ axis is used here to define longitudinal and transverse phonon vibrations). The LO-TO splitting mainly occurs for the highfrequency optical branches of $\mathrm{LO}_{1}$ and $\mathrm{TO}_{1,2}$. c Schematic illustration of the screening effect on the LO-TO splitting (following the Eqs. (2) and (4) in Methods) with different Thomas-Fermi screening lengths, $r_{\mathrm{TF}}$ (in units of the lattice constant). With enhanced screening or smaller $r_{\mathrm{TF}}$ as traced by the arrow, the LO frequency around the Brillouin zone center is suppressed. The Born effective charge, $\mathcal{Z}_{a}$, is supposed to be insensitive to the presence of free carriers, for this purpose, which is an approximation. d Comparison of the neutron-weighted phonon DOSs between $\mathrm{ZrNiSn}$ and $\mathrm{ZrNiSn}_{0.875} \mathrm{Sb}_{0.125}$ calculated with first principles. e Variation of the LO-TO splitting (or polarization field) with $n$. The dash curve is fitted using the Eqs. (2) and (4) in Methods including screening. The solid curve is the $n$-dependent mobility limited by polar optical phonon scattering in $\mathrm{ZrNiSn}_{1-x} \mathrm{Sb}_{x} \mathrm{compounds}^{40}$. With increasing screening, the LO-TO splitting and polarization field are reduced, and the polar optical phonon scattering is reduced. $\mathbf{f}$ The polar coupling constant for some selected TE semiconductors as listed in Fig. 1. The value for the half-Heusler compounds is calculated based on the parameters as shown in Supplementary Table 1, while the data for other compounds is from literature ${ }^{27}$.

carrier transport, via the Fröhlich electron-phonon interaction $^{27,52}$. This coupling between electrons and polar optical phonons can be assessed by the dimensionless polar coupling constant:

$$
\alpha_{\mathrm{PO}}=\frac{e^{2}}{4 \pi \hbar}\left(\frac{m^{*}}{2 \hbar \omega_{\mathrm{LO}}}\right)^{1 / 2}\left(\frac{1}{\varepsilon_{\infty}}-\frac{1}{\varepsilon_{\mathrm{s}}}\right)
$$

$\hbar$ is the Planck constant, $m^{*}$ is the effective mass of electrons, $\omega_{\mathrm{LO}}$ is the LO frequency, and $\varepsilon_{\infty}$ and $\varepsilon_{s}$ are the high-frequency and static dielectric functions. The observation of the LO-TO splitting in $\mathrm{ZrNiSn}$ confirms that $\mathrm{HH}$ compounds exhibit polar chemical bonds ${ }^{53}$. This mixed bonding can also be important for the thermal conductivity ${ }^{54}$. The polar coupling constant, $\alpha_{\mathrm{PO}}$, for $\mathrm{ZrNiSn}$ is estimated as 0.32 , indicating that the polar optical phonon scattering could play an important role in electrical transport properties.

We now turn to the collapse of the tail in the phonon DOSs with Sb doping (Fig. 3b). The optical phonon energy is connected to the reduced-atomic-mass by the relation: $\omega_{\mathrm{op}} \propto M_{r}^{-1 / 2}$, where 
the reduced-atomic-mass is defined as $M_{\mathrm{r}}=1 / \sum_{a}\left(1 / M_{a}\right)$, where $M_{a}$ is the mass of atom $a$ in unit cell. However, the modification of the optical phonon energy by $12 \%$ doping of $\mathrm{Sb}$ is less than $0.04 \%$, which is too small to explain the change observed in the phonon DOSs.

Instead, the screening effect needs to be considered to explain the collapse of the LO-TO splitting. The presence of free carriers and the concomitant screening can modify both the electron-LO phonon interaction and the dispersion of the LO optical phonons, such as Kohn anomaly of the phonon branch in metals ${ }^{55}$. For the LO-TO splitting, the additional restoring force of the LO vibrations is a Coulomb interaction. This Coulomb interaction can be well screened by free carriers. This can be described in the Thomas-Fermi approximation (see methods for details) ${ }^{56}$. Higher free carrier concentration leads to better screening, shorter Thomas-Fermi screening length, $r_{\mathrm{TF}}$, and hence smaller Coulomb interaction and LO-TO splitting as schematized in Fig. 4c. The phonon DOSs (in Fig. 4d) from first-principles calculations with consideration of the screening effect reproduce this change (detailed changes in the phonon dispersion with Sb doping are given in Supplementary Fig. 6). This change is also confirmed by the reduced LO-TO splitting as determined from the phonon DOSs measurements (see Fig. 4e). This reduction of LO-TO splitting could also be well simulated (dashed line in Fig. 4e) with the screening model as described with Eqs. (2) and (4) in Methods. In addition, this reduced LO-TO splitting or polarization field is accompanied by a raising mobility, $\mu_{\text {po }}(n)$, that is limited by polar optical phonon scattering ${ }^{40}$. However, the release of the polar optical phonon scattering is accompanied by an enhancement of the acoustic phonon scattering as shown in Fig. 2c. This crossover lead to an optimal carrier concentration and mobility around $n=3-5 \times 10^{20} \mathrm{~cm}^{-3}$ for the TE performance of $\mathrm{ZrNiSn}$-based compounds (Fig. 2c).

Following the screening scenario, additional free charge carriers could enhance the screening effect in all semiconductors, and transitions between different carrier scattering mechanisms are expected from the low $n / p$ region to the high $n / p$ region, hence a non-monotonous carrier mobility. However, of the semiconductors listed in Fig. 1, only the lead chalcogenides and half-Heusler compounds are known to exhibit non-monotonous behavior at room temperature. To explain the different dependences of carrier mobility on carrier concentration, the polar coupling constants of the half-Heusler compounds are calculated using the Eq. (1) (see Supplementary Table 1 and Supplementary Fig. 7 for details). A comparison of the polar coupling constants between different samples is shown in Fig. 4f. Compared with the lead chalcogenides and half-Heusler compounds, the polar coupling is much weaker in $\mathrm{InSb}, \mathrm{CoSb}_{3}$, and GaAs compounds, indicating smaller, and perhaps negligible polar optical phonon scattering in those compounds. Therefore, screening of the polar optical phonon scattering does not make important changes to the carrier mobility in those compounds. In contrast, the screening of polar optical phonon scattering in the more strongly-polar lead chalcogenides and half-Heusler compounds would noticeably tune the dominant carrier scattering mechanisms, leading to the non-monotonous behavior.

Finally, we comment on the details of the phonon modes based on first-principles calculations. The results are shown in the insets of Fig. $4 \mathrm{~b}$. The primitive unit cell of $\mathrm{ZrNiSn}$ contains three atoms, and therefore, there are 6 optical phonon branches. The three high energy optical phonon branches correspond to the LO-TO splitting, while the other three low energy optical phonon branches do not present obvious LO-TO splitting. For both the high and low energy branches, the $\mathrm{Ni}$ and $\mathrm{Sn}$ atoms vibrate in opposite directions. This is consistent with the molecular orbitals analysis of $\mathrm{ZrNiSn}$, where the $\mathrm{Ni}$ and $\mathrm{Sn}$ atoms primarily form $(\mathrm{NiSn})^{4-}$ clusters and then bond with the $\mathrm{Zr}^{4+}$ cations to form the $\mathrm{ZrNiSn}$ compound ${ }^{57}$. The difference between the high and low energy branches relates to the vibration of $\mathrm{Zr}$. Given that the $\mathrm{Ni}$ atoms sit at the centers of both the $\mathrm{Zr}$ and Sn tetrahedrons, the opposite vibration of both the $\mathrm{Zr}$ and $\mathrm{Sn}$ with respect to the $\mathrm{Ni}$ would involve relatively larger chemical bonding forces, and hence correspond to the higher energy optical phonon branches. The small LO-TO splitting for the low energy optical phonon can then be attributed to the relatively larger group velocity and small displacement of the $(\mathrm{NiSn})^{4-}$ cluster with respect to the $\mathrm{Zr}$ sublattice.

\section{Discussion}

The effects of dielectric screening on carrier mobility are not just limited to the polar optical phonon scattering ${ }^{58,59}$. Rather we find other important effects. Polar optical phonon scattering becomes weak at low temperature, because of the Bose factor that means that there are no excited optical phonons at low temperature. However, the non-monotonous behavior of the $\mu(n / p)$ does not disappear at low temperature, but becomes more evident as depicted in Supplementary Fig. 8. This phenomenon is attributed to the screening of the ionized impurity and grain boundary scatterings, since these are the dominant scattering mechanisms at low temperatures. Taken the ionized impurity scattering as an example, the potential of the ionized impurities in heavily doped semiconductor cannot be described by the bare Coulomb potential, $\varphi(r) \propto \frac{1}{r}$, any more, while a new formula in the Thomas-Fermi approximation, $\varphi(r) \propto \frac{1}{r} \exp \left(-\frac{r}{r_{\mathrm{TF}}}\right)$, should be applied $^{56}$. As schematized in Supplementary Fig. 9 , the screened potential drops more quickly than the bare Coulomb potential, and then the scattering is weaker.

According to the definition of the Thomas-Fermi screening length (see Eq. (5) in Methods), the screening effect becomes weaker with increasing temperature. Nonetheless, it is still effective at higher temperature, even at $800 \mathrm{~K}^{40}$. This is important for the manipulation of the carrier scattering mechanisms in the ZrNiSn-based compounds. In addition, our previous study demonstrated that the polar optical phonon, acoustic phonon, and alloy scatterings, which are the main carrier scattering mechanisms at $300 \mathrm{~K}$, still play the dominated roles at higher temperature, and crossover between these scattering mechanisms can also been realized by introducing the screening effect ${ }^{40}$.

In summary, different carrier scattering mechanisms in ZrNiSn-based half-Heusler compounds are studied with highquality single and polycrystalline crystals, combined with electrical transport measurements, neutron powder diffraction and inelastic neutron scattering measurements, as well as theoretical calculations. With increasing carrier concentration $n$, a crossover of the carrier mobility $\mu(n)$ and optimal TE performance are found in the region of $n=3-5 \times 10^{20} \mathrm{~cm}^{-3}$. This crossover of $\mu$ (n) corresponds to the variation of the dominant scattering mechanisms, from ionized impurity and grain boundary scattering to acoustic phonon/alloy scatterings at low temperature, or from polar optical phonon scattering to acoustic phonon/alloy scatterings at high temperature. These crossovers between different dominant scattering mechanisms are realized through the screening of the ionized impurity, grain boundary and polar optical phonon scattering, and the enhancement of acoustic phonon/alloy scatterings. On the bases of these insights into the different carrier scatterings, a carrier scattering phase diagram may be derived. This is shown in Fig. 5, and could be used as a guide for the further improvement of the TE properties in the half-Heusler TE materials. For example, grain boundaries, which are frequently employed to reduce lattice thermal conductivity, 
a

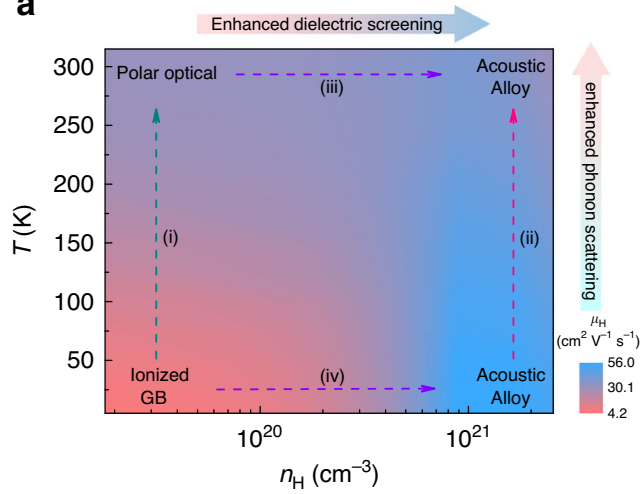

b

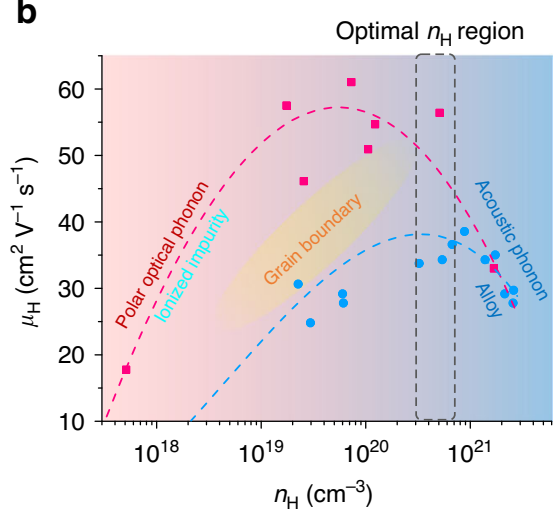

Fig. 5 Carrier scattering phase diagram for ZrNiSn-based half-Heusler compounds. a The phase diagram as functions of temperature and carrier concentration. GB represent grain boundary scattering. $\mathbf{b}$ The phase diagram with single-crystalline and polycrystalline data as function of carrier concentration at $300 \mathrm{~K}$. The dash lines in the figures are guides of the eye.

have an adverse effect on carrier transport in lightly doped halfHeusler compounds, e.g. $\mathrm{NbFe}_{0.95} \mathrm{Ti}_{0.05} \mathrm{Sb}^{60}$. This would work against the efforts to suppress the lattice thermal conductivity in improving TE performance. However, the grain boundary scattering for carrier transport could be well screened in heavily doped semiconductor, e.g. $\mathrm{NbFe}_{0.8} \mathrm{Ti}_{0.2} \mathrm{Sb}$, and the introduction of grain boundaries could successfully improve the TE performance ${ }^{49}$. We expect the establishment of such a carrier scattering phase diagram in other TE systems could also help to choose reasonable and effective strategies for the optimization of TE performance.

\section{Methods}

Synthesis. Ingots of nominal $\mathrm{ZrNiSn}_{1-x} \mathrm{Sb}_{x}(x=0.00,0.04,0.08$, and 0.12$)$ compounds were synthesized by levitation melting of stoichiometric amount of $\mathrm{Zr}, \mathrm{Ni}$, $\mathrm{Sn}$, and $\mathrm{Sb}$ under argon atmosphere. The melting was carried out three times to ensure homogeneity. The ingots were ground into powder, and then sintered using spark plasma sintering (SPS-1050, Sumitomo Coal Mining Co.) at $1193 \mathrm{~K}$ for $10 \mathrm{~min}$ under a pressure of $65 \mathrm{MPa}$ in vacuum. The densities of the as-sintered samples are $95 \%$ of theoretical densities. Then, the SPS-samples were used for both physical properties and neutron scattering measurements. NPD measurements (Supplementary Fig. 1) do not show discernible impurity phases. All samples show extra $\mathrm{Ni}$ at the vacancy site, and the occupancy varies between $4.9 \%$ and $6.4 \%$, being consistent with previous results from Electron Probe Microanalysis measurements ${ }^{40}$

The undoped and $\mathrm{Sb}$-doped $\mathrm{ZrNiSn}_{1-y} \mathrm{Sb}_{y}$ single crystals were grown by a $\mathrm{Sn}$ Flux method. The starting powders of $\mathrm{Zr}, \mathrm{Ni}, \mathrm{Sn}$, and $\mathrm{Sb}$ were mixed together in a molar ratio of 1: 1: 10: $y(y=0-0.10)$. The mixtures were sealed in a dry quartz tube under high vacuum, which was heated up to $1100^{\circ} \mathrm{C}$ in $15 \mathrm{~h}$ and further dwelled for $24 \mathrm{~h}$. After that, the tube was slowly cooled down to $650^{\circ} \mathrm{C}$ at a rate of $2{ }^{\circ} \mathrm{C} / \mathrm{h}$. Single crystals were obtained after a centrifuging process to remove the $\mathrm{Sn}$ flux. The as-grown single crystals with typical size of $2 \mathrm{~mm}$ were checked and oriented at room temperature by a Laue X-ray diffractometer, which show sharp and distinct diffraction spots (Supplementary Fig. 2), indicating high quality and crystallinity.

Physical properties. The electrical and thermal transport properties are characterized using the Physical Property Measurement System (PPMS) with the electrical transport option and thermal transport options. To keep consistent, the electrical resistivity was measured along [110] direction for all the single crystals, while the magnetic field was applied along [11ㅣ direction during the Hall resistivity measurement. Hall carrier concentration $n_{\mathrm{H}}$ was calculated using the equation $n_{\mathrm{H}}=1 /\left(e R_{\mathrm{H}}\right)$, where $e$ is the unit charge and $R_{\mathrm{H}}$ is the Hall coefficient. The carrier mobility $\mu_{\mathrm{H}}$ was calculated using $\mu_{\mathrm{H}}=R_{\mathrm{H}} / \rho$. The Lorenz number is calculated by the single parabolic band model ${ }^{12}$.

Neutron powder diffraction. Approximately 3 grams samples were ground into powder in agate mortar and used for NPD measurements. The measurements were taken on the Super High Resolution Powder Diffractometer, BL08 SuperHRPD, at the Material and Life Science Experimental Facility (MLF) of Japan Proton Accelerator Research Complex (J-PARC) and on the General Purpose Powder Diffractometer, GPPD, at the China Spallation Neutron Source. Vanadium sample can were used in the measurements. The NPD patterns were collected over the temperature range of $\sim 10 \mathrm{~K}$ to $\sim 300 \mathrm{~K}$. Rietveld refinement were performed using the Z-Rietveld software.

Inelastic neutron scattering. The inelastic neutron scattering measurements were performed using the High Resolution Chopper Spectrometer, BL12 HRC, at MFL of J-PARC. Approximately 13 grams of powder samples were wrapped in aluminum foil and then sealed in thin-walled cylindrical Al can, filled with low-pressure helium gas. The incident neutron energies, $E_{\mathrm{i}}=66 \mathrm{meV}$, was used with an energy resolution (full width at half maximum) of $\Delta E / E_{\mathrm{i}} \sim 5 \%$. The measurements were performed at $10 \mathrm{~K}, 100 \mathrm{~K}, 200 \mathrm{~K}$, and $300 \mathrm{~K}$ with a closed-cycle He refrigerator. Correspondingly, the empty $\mathrm{Al}$ can was also measured in identical conditions at all temperatures. All of the spectra were normalized with respect to the total incident flux. The time-of-flight data were reduced with the HANA (a program for data reduction collected on HRC). Then, the obtained powder-averaged dynamical structure factor $S(\mathbf{Q}, E)$ were analyzed in the incoherent-scattering approximation after substracting background, multiphonon and multiple scattering as well as the elastic peak using the $\mathrm{DAVE}^{61}$ and GetDOS programs ${ }^{62}$. Integration of the $S(\mathbf{Q}, E)$ spectra over the range of momentum transfers $0.4 \leq \mathbf{Q} \leq 6.0 \AA^{-1}$ leads to neutronweighted phonon DOSs for the samples.

First-principles calculations. The ab initio calculations were performed by using the projector augmented wave (PAW) method, as implemented in the Vienna ab initio simulation package (VASP) ${ }^{63,64}$. The generalized gradient approximation (GGA) was used for the exchange-correlation functional ${ }^{65}$, and a plane-wave energy cutoff of $450 \mathrm{eV}$ was adopted. The phonon DOS and dispersions of $\mathrm{ZrNiSn}$ and $\mathrm{Sb}$-doped $\mathrm{ZrNiSn}$ were calculated by using the frozen phonon method, as implemented in the Phonopy package ${ }^{66}$. The phonon group velocities, the scattering rates, and lattice thermal conductivities were obtained via the shengBTE package, in which we considered the interactions between atoms to their fourth nearest neighbors ${ }^{67}$. A $4 \times 4 \times 4$ supercell (192 atoms) was used in both procedures. For the structural optimization of the unit cell, the k-points was chosen as $8 \times 8 \times 8$, and the convergence accuracy of force was $10^{-5} \mathrm{eV} / \AA$. For the supercells with displacements, only $\Gamma$ point was considered. The energy convergence criterion was $10^{-7} \mathrm{eV}$ throughout the work.

Physical model of LO-TO splitting and screening effect. The frequency (energy) difference between LO and TO arises from the long-range electric field generated by ionic polarization with the LO phonon vibration. Following the physical model proposed by Born and Huang ${ }^{51}$, the relation between LO and TO is described as ${ }^{68}$ :

$$
\omega_{\mathrm{LO}}^{2}=\omega_{\mathrm{TO}}^{2}+\Psi(\mathbf{q}) \frac{|\mathbf{q}|^{2}}{\Omega}\left(\sum_{a} \frac{\mathbf{e}_{\mathbf{q}} \cdot \mathcal{Z}_{a} \cdot \mathbf{e}_{\mathrm{LO}}^{a}}{\sqrt{M_{a}}}\right)^{2}
$$

where $\omega_{\mathrm{LO}}$ and $\omega_{\mathrm{TO}}$ are the frequencies of LO and TO, $\mathbf{e}_{\mathbf{q}}$ is the unit vector along the momentum direction of $\mathbf{q}, \mathbf{e}_{\mathrm{LO}}^{a}$ is the $|\mathbf{q}| \rightarrow 0$ limit of the eigenvector of the dynamical matrix of the LO mode, $M_{a}$ is the mass of atom $a$ in unit cell, $\mathcal{Z}_{a}$ is the Born effective charge of atom $a$, which reflect the change in polarization in response to the atom displacement, and $\Psi(\mathbf{q})$ is the Coulomb interaction in Fourier space, defined as:

$$
\Psi(\mathbf{q})=\frac{e^{2}}{\varepsilon_{\infty}|\mathbf{q}|^{2}}
$$

where $\varepsilon_{\infty}$ is the high-frequency (optical) dielectric function. In the limit of $|\mathbf{q}| \rightarrow 0$, the Eq. (1) could be simply replaced by the Lyddane-Sachs-Teller relationship: $\omega_{\mathrm{LO}}^{2} / \omega_{\mathrm{TO}}^{2}=\varepsilon_{\mathrm{s}} / \varepsilon_{\infty}, \varepsilon_{\mathrm{s}}$ is the static dielectric function ${ }^{69}$. 
Presence of free carriers and the concomitant screening effect can modify both the electron-LO phonon interaction and the dispersion characteristics of the LO optical phonons. Then, the Coulomb interaction in the momentum space is modified by a factor of $|\mathbf{q}|^{2} r_{T F}^{2} /\left(1+|\mathbf{q}|^{2} r_{T F}^{2}\right)$ according to the Thomas-Fermi approximation, and then the Eq. (3) can be rewritten as:

$$
\Psi(\mathbf{q})=\frac{e^{2} r_{T F}^{2}}{\varepsilon_{\infty}\left(1+|\mathbf{q}|^{2} r_{T F}^{2}\right)}
$$

Where $r_{\mathrm{TF}}$ is the Thomas-Fermi screening length (or Debye length), and is defined as:

$$
r_{\mathrm{TF}}=\sqrt{\frac{\varepsilon k_{\mathrm{B}} T}{n e^{2}}}
$$

Where $k_{\mathrm{B}}$ is the Boltzmann constant. Higher $n$ leads to smaller $r_{\mathrm{TF}}$, and smaller $r_{\mathrm{TF}}$ means better screening effect. Enhanced screening effect leads to a smaller modified Coulomb interaction, and hence smaller LO-TO splitting as schematized in Fig. 4c.

It is necessary to point out that the screening model used here is in a static (Thomas-Fermi) approximation. It assumes that the scattering potential is static, or charge carriers are fast enough in response to movement of scattering potential, so that a screening scenario could form. This assumption is valid for static ionized impurity and grain boundary scatterings. However, it become more complex to deal with the polar optical phonon scattering. If the plasma (collective oscillation of free charges) frequency, $\omega_{\mathrm{P}}=\sqrt{n e^{2} / \varepsilon_{\infty} m^{*}}$, is smaller than the optical phonon frequency $\omega_{\mathrm{op}}$, the charge carriers lag behind the lattice vibration, which produces an anti-screening effect, and enhances the LO-TO splitting and the polar optical phonon scattering 58,70 . Only when $\omega_{\mathrm{P}}>\omega$, can the charge carriers instantaneously respond to the lattice vibration, and then meets the prerequisite for the approximation of static screening ${ }^{58,70}$. In the case of $\mathrm{ZrNiSn}$-based half-Heusler compounds, $\omega_{\mathrm{P}}$ is estimated as $64.28 \mathrm{THz}$ when $n=1 \times 10^{20} \mathrm{~cm}^{-3}$ (the highfrequency dielectric parameter was calculated as $\varepsilon_{\infty}=22.07$ and the calculated effective mass of electron $m^{*}=2.8 \pm 0.2 m_{\mathrm{e}}$ are used here $\left.{ }^{40}\right)$. This value of $\omega_{\mathrm{P}}$ is much larger than frequency of the longitudinal optical phonon, $\sim 7.25 \mathrm{THz}$ (or $\sim 30 \mathrm{meV}$ ), and the Thomas-Fermi screening model is valid in the analysis of the screening of polar optical phonon scattering here.

\section{Data availability}

The data that support the findings of this study are available from the corresponding authors on reasonable request.

Received: 1 March 2020; Accepted: 2 June 2020;

Published online: 19 June 2020

\section{References}

1. Bell, L. E. Cooling, heating, generating power, and Recovering Waste Heat with Thermoelectric Systems. Science 321, 1457-1461 (2008).

2. Appel, O. \& Gelbstein, Y. A comparison between the effects of $\mathrm{Sb}$ and $\mathrm{Bi}$ Doping on the thermoelectric properties of the $\mathrm{Ti}_{0.3} \mathrm{Zr}_{0.35} \mathrm{Hf}_{0.35} \mathrm{NiSn}$ halfHeusler alloy. J. Electron. Mater. 43, 1976-1982 (2013).

3. Guttmann, G. M., Dadon, D. \& Gelbstein, Y. Electronic tuning of the transport properties of off-stoichiometric $\mathrm{Pb}_{\mathrm{x}} \mathrm{Sn}_{1-\mathrm{x}} \mathrm{Te}$ thermoelectric alloys by $\mathrm{Bi}_{2} \mathrm{Te}_{3}$ doping. J. Appl. Phys. 118, 065102 (2015).

4. Komisarchik, G., Fuks, D. \& Gelbstein, Y. High thermoelectric potential of $\mathrm{n}$ type $\mathrm{Pb}_{1-\mathrm{x}} \mathrm{Ti}_{\mathrm{x}} \mathrm{Te}$ alloys. J. Appl. Phys. 120, 055104 (2016).

5. Cohen, I., Kaller, M., Komisarchik, G., Fuks, D. \& Gelbstein, Y. Enhancement of the thermoelectric properties of $\mathrm{n}$-type $\mathrm{PbTe}$ by $\mathrm{Na}$ and $\mathrm{Cl}$ co-doping. $J$. Mater. Chem. C 3, 9559-9564 (2015).

6. Sadia, Y., Aminov, Z., Mogilyansky, D. \& Gelbstein, Y. Texture anisotropy of higher manganese silicide following arc-melting and hot-pressing. Intermetallics 68, 71-77 (2016).

7. Tang, Y. et al. Convergence of multi-valley bands as the electronic origin of high thermoelectric performance in $\mathrm{CoSb}_{3}$ skutterudites. Nat. Mater. 14, 1223-1228 (2015).

8. Imasato, $\mathrm{K}$. et al. Metallic n-type $\mathrm{Mg}_{3} \mathrm{Sb}_{2}$ single crystals demonstrate the absence of ionized impurity scattering and enhanced thermoelectric performance. Adv. Mater. 32, 1908218 (2020).

9. Nolas G. S., Sharp J., Goldsmid J. Thermoelectrics: Basic Principles And New Materials Developments (Springer Science \& Business Media, 2013).

10. Rowe D. M. CRC handbook of Thermoelectrics (CRC press, 1995).

11. Snyder, G. J. \& Toberer, E. S. Complex thermoelectric materials. Nat. Mater. 7, 105-114 (2008).

12. Xie, H. et al. Beneficial contribution of alloy disorder to electron and phonon transport in half-Heusler thermoelectric materials. Adv. Funct. Mater. 23, 5123-5130 (2013).
13. Wang, S. et al. On intensifying carrier impurity scattering to enhance thermoelectric performance in $\mathrm{Cr}$-Doped $\mathrm{Ce}_{\mathrm{y}} \mathrm{Co}_{4} \mathrm{Sb}_{12}$. Adv. Funct. Mater. 25, 6660-6670 (2015)

14. Fischetti M. V., Vandenberghe W. G. Advanced Physics Of Electron Transport In Semiconductors And Nanostructures. (Springer, 2016).

15. Wang, H., Pei, Y., LaLonde, A. D. \& Snyder, G. J. Weak electron-phonon coupling contributing to high thermoelectric performance in n-type PbSe. Proc. Natl Acad. Sci. USA 109, 9705 (2012).

16. Delaire, O. et al. Giant anharmonic phonon scattering in $\mathrm{PbTe}$. Nat. Mater. 10, 614-619 (2011).

17. Shiga, T. et al. Microscopic mechanism of low thermal conductivity in lead telluride. Phys. Rev. B 85, 155203 (2012).

18. Li, C. W. et al. Phonon self-energy and origin of anomalous neutron scattering spectra in SnTe and PbTe thermoelectrics. Phys. Rev. Lett. 112, 175501 (2014).

19. Christensen, M. et al. Avoided crossing of rattler modes in thermoelectric materials. Nat. Mater. 7, 811-815 (2008).

20. Lan, Y., Minnich, A. J., Chen, G. \& Ren, Z. Enhancement of thermoelectric figure-of-merit by a bulk nanostructuring approach. Adv. Funct. Mater. 20, 357-376 (2010).

21. Ma, J. et al. Glass-like phonon scattering from a spontaneous nanostructure in $\mathrm{AgSbTe}_{2}$. Nat. Nanotechnol. 8, 445 (2013).

22. Ma, J. et al. Phonon scattering rates and atomic ordering in $\mathrm{Ag}_{1-\mathrm{x}} \mathrm{Sb}_{1+\mathrm{x}} \mathrm{Te}_{2+\mathrm{x}}$ $(x=0,0.1,0.2)$ investigated with inelastic neutron scattering and synchrotron diffraction. Phys. Rev. B 90, 134303 (2014).

23. $\mathrm{Li}, \mathrm{B}$. et al. Liquid-like thermal conduction in intercalated layered crystalline solids. Nat. Mater. 17, 226-230 (2018).

24. Peter Y., Cardona M. Fundamentals Of Semiconductors: Physics And Materials Properties (Springer Science \& Business Media, 2010)

25. LaBotz, R. J. \& Mason, D. R. The thermal conductivities of $\mathrm{Mg}_{2} \mathrm{Si}$ and $\mathrm{Mg}_{2} \mathrm{Ge}$. J. Electrochem. Soc. 110, 121-126 (1963).

26. Klemens, P. G. Thermal resistance due to point defects at high temperatures. Phys. Rev. 119, 507-509 (1960).

27. Kōmoto K., Mori T. Thermoelectric Nanomaterials: Materials Design and Applications. (Springer, 2013).

28. Wang, H., Schechtel, E., Pei, Y. \& Snyder, G. J. High thermoelectric efficiency of n-type PbS. Adv. Energy Mater. 3, 488-495 (2013).

29. Zawadzki, W. Electron transport phenomena in small-gap semiconductors. Adv. Phys. 23, 435-522 (1974).

30. Caillat, T., Borshchevsky, A. \& Fleurial, J. P. Properties of single crystalline semiconducting $\mathrm{CoSb}_{3}$. J. Appl. Phys. 80, 4442-4449 (1996).

31. Levinshtein M., Rumyantsev S., Shur M. Handbook Series on Semiconductor Parameters (Word Scientific, 1996).

32. Sun, H. L., Yang, C. L., Wang, M. S. \& Ma, X. G. Remarkably high thermoelectric efficiencies of the half-Heusler compounds BXGa (X $=\mathrm{Be}, \mathrm{Mg}$, and Ca). ACS Appl. Mater. Interfaces 12, 5838-5846 (2020).

33. $\mathrm{Fu}, \mathrm{C}$. et al. Realizing high figure of merit in heavy-band p-type half-Heusler thermoelectric materials. Nat. Commun. 6, 8144 (2015).

34. Xing, Y. et al. High-efficiency half-Heusler thermoelectric modules enabled by self-propagating synthesis and topologic structure optimization. Energy Environ. Sci. 12, 3390-3399 (2019).

35. Aliev, F. G. Gap at Fermi level in some new d- and f-electron intermetallic compounds. Phys. B 171, 199-205 (1991).

36. Aliev, F. G., Kozyrkov, V. V., Moshchalkov, V. V., Scolozdra, R. V. \& Durczewski, K. Narrow band in the intermetallic compounds MNiSn $(\mathrm{M}=\mathrm{Ti}$, Zr, Hf). Z. Phys. B Condens. Matter 80, 353-357 (1990).

37. Uher, C., Yang, J., Hu, S., Morelli, D. T. \& Meisner, G. P. Transport properties of pure and doped MNiSn (M=Zr, Hf). Phys. Rev. B 59, 8615-8621 (1999).

38. Joshi, G. et al. Enhancement in thermoelectric figure-of-merit of an N-type half-Heusler compound by the nanocomposite approach. Adv. Energy Mater. 1, 643-647 (2011).

39. Yu, J. et al. Unique role of refractory Ta alloying in enhancing the figure of merit of NbFeSb thermoelectric materials. Adv. Energy Mater. 8, 1701313 (2018).

40. Xie, H. et al. The intrinsic disorder related alloy scattering in ZrNiSn halfHeusler thermoelectric materials. Sci. Rep. 4, 6888 (2014).

41. Fu, C., Zhu, T., Liu, Y., Xie, H. \& Zhao, X. Band engineering of high performance p-type FeNbSb based half-Heusler thermoelectric materials for figure of merit zT > 1. Energy Environ. Sci. 8, 216-220 (2015).

42. Qiu, Q. et al. Grain boundary scattering of charge transport in n-type (Hf,Zr) CoSb half-Heusler thermoelectric Materials. Adv. Energy Mater. 9, 1803447 (2019).

43. Sekimoto, T., Kurosaki, K., Muta, H. \& Yamanaka, S. High-thermoelectric figure of merit realized in p-type half-Heusler compounds: $\mathrm{ZrCoSn}_{\mathrm{x}} \mathrm{Sb}_{1-\mathrm{x}} \cdot \mathrm{Jpn}$ J. Appl. Phys. 46, L673-L675 (2007).

44. Shuai, J. et al. Tuning the carrier scattering mechanism to effectively improve the thermoelectric properties. Energy Environ. Sci. 10, 799-807 (2017).

45. Kuo, J. J. et al. Grain boundary dominated charge transport in $\mathrm{Mg}_{3} \mathrm{Sb}_{2}$-based compounds. Energy Environ. Sci. 11, 429-434 (2018). 
46. Fu, C. et al. Revealing the intrinsic electronic structure of 3D half-Heusler thermoelectric materials by angle-resolved photoemission spectroscopy. Adv. Sci. 7, 1902409 (2020).

47. Chen, S. et al. Thermal conductivity of isotopically modified graphene. Nat. Mater. 11, 203-207 (2012)

48. Lindsay, L., Broido, D. A. \& Reinecke, T. L. Phonon-isotope scattering and thermal conductivity in materials with a large isotope effect: A first-principles study. Phys. Rev. B 88, 144306 (2013).

49. $\mathrm{Fu}, \mathrm{C}$. et al. Enhancing the figure of merit of heavy-band thermoelectric materials through hierarchical phonon scattering. Adv. Sci. 3, 1600035 (2016).

50. Zhou, J. et al. Large thermoelectric power factor from crystal symmetryprotected non-bonding orbital in half-Heuslers. Nat. Commun. 9, 1721 (2018).

51. Born M., Huang K. Dynamical Theory Of Crystal Lattices. (Clarendon press, 1954).

52. Ahn, D. Theory of polar-optical-phonon scattering in a semiconductor quantum wire. J. Appl. Phys. 69, 3596-3600 (1991).

53. Kandpal, H. C., Felser, C. \& Seshadri, R. Covalent bonding and the nature of band gaps in some half-Heusler compounds. J. Phys. D Appl. Phys. 39, 776-785 (2006).

54. Feng, Z., Fu, Y., Zhang, Y. \& Singh, D. J. Characterization of rattling in relation to thermal conductivity: ordered half-Heusler semiconductors. Phys. Rev. B 101, 064301 (2020).

55. Kohn, W. Image of the Fermi Surface in the vibration spectrum of a metal. Phys. Rev. Lett. 2, 393-394 (1959).

56. Singh J. Electronic And Optoelectronic Properties Of Semiconductor Structures (Cambridge University Press, 2007).

57. Zeier, W. G. et al. Engineering half-Heusler thermoelectric materials using Zintl chemistry. Nat. Rev. Mater. 1, 16032 (2016).

58. Ridley B. K. Quantum Processes In Semiconductors (Oxford University Press, 2013).

59. Wang, Y. et al. Doubling the ZT record of $\mathrm{TiS}_{2}$-based thermoelectrics by incorporation of ionized impurity scattering. J. Mater. Chem. C 6, 9345-9353 (2018).

60. He, R. et al. Achieving high power factor and output power density in p-type half-Heuslers $\mathrm{Nb}_{1-\mathrm{x}} \mathrm{Ti}_{\mathrm{x}} \mathrm{FeSb}$. Proc. Natl Acad. Sci. USA 113, 13576-13581 (2016).

61. Azuah, R. T. et al. DAVE: a comprehensive software suite for the reduction, visualization, and analysis of low energy neutron spectroscopic data. J. Res. Natl Inst. Stand. Technol. 114, 341-358 (2009).

62. Lin, Y., Islam, F. \& Kresh, M. Multiphonon: phonon density of states tools for inelastic neutron scattering powder data. J. Open Source Softw. 3, 440 (2018).

63. Kresse, G. \& Furthmüller, J. Efficient iterative schemes for ab initio total-energy calculations using a plane-wave basis set. Phys. Rev. B 54, 11169-11186 (1996).

64. Blöchl, P. E. Projector augmented-wave method. Phys. Rev. B 50, 17953-17979 (1994).

65. Perdew, J. P., Burke, K. \& Ernzerhof, M. Generalized gradient approximation made simple. Phys. Rev. Lett. 77, 3865-3868 (1996).

66. Togo, A. \& Tanaka, I. First principles phonon calculations in materials science. Scr. Mater. 108, 1-5 (2015).

67. Li, W., Carrete, J. A., Katcho, N. \& Mingo, N. ShengBTE: a solver of the Boltzmann transport equation for phonons. Comput. Phys. Commun. 185, 1747-1758 (2014).

68. Sohier, T., Gibertini, M., Calandra, M., Mauri, F. \& Marzari, N. Breakdown of optical phonons' splitting in two-dimensional materials. Nano Lett. 17, 3758-3763 (2017).

69. Lyddane, R. H., Sachs, R. G. \& Teller, E. On the polar vibrations of alkal halides. Phys. Rev. 59, 673-676 (1941).

70. Ehrenreich, H. Screening effects in polar semiconductors. J. Phys. Chem. Solids 8, 130-135 (1959).

\section{Acknowledgements}

J.M. thanks the financial support from the National Science Foundation of China (No. 11774223 and No. U1732154) and a Shanghai talent program. C.G.F. thanks the financial support from the Deutsche Forschungsgemeinschaft (DFG, German Research Foundation) - Projektnummer (No. 392228380) and the Alexander von Humboldt Foundation J.Y. acknowledges the financial support from Natural Science Foundation of China (No. 11674211 and No. 51761135127), and the 111 project D16002. T.J.Z. thanks the financial support from the National Science Foundation of China (No. 51761135127) and the National Science Fund for Distinguished Young Scholars (No. 51725102). Q.R. thanks Dr. Jiao Lin from the Oak Ridge National Lab for the help on the phonon DOSs analysis using the GetDOS programs, and Dr. Xiaokun Gu for the discussion about LO-TO splitting. The inelastic neutron scattering data using HRC were supported with the general proposal (Proposal No. 2017A0071 and 2018B0281) of J-PARC/MLF. Some of the neutron diffraction data using SuperHRPD were taken under both the general proposal (Proposal No. 2017A0118) and the fast track proposal (Proposal No. 2017BF0804) of J-PARC/MLF, while some data using GPPD were taken under the proposal (Proposal No. P2018091800008) of CSNS.

\section{Author contributions}

Q.R., Z.L., T.M., S.A., and J.M. designed and performed the INS experiment using HRC at the J-PARC. Q.R., M.H., S.L., S.T., T.K., and J.M. designed and performed the highresolution neutron diffraction experiment using superHRPD at the J-PARC. Q.R., L.H., X.T., and J. M. designed and performed the neutron diffraction experiment using GPPD at the CSNS. C.G.F. and Q.Q. synthesized the samples. C.G.F. and C.F. performed the transport properties measurement. Q.R. analyzed the experiment data, along with all coauthors. J.Y. and S.D. performed the First-principles calculations. Q.R., J.Y., D.J.S., and J.M. interpreted the LO-TO splitting phenomenon. Q.R., C.G.F., T.Z., J.Y. and J.M. convinced the project and drafted the manuscript. All authors analyzed and reviewed the results, and provided input to this paper.

\section{Competing interests}

The authors declare no competing interests.

\section{Additional information}

Supplementary information is available for this paper at https://doi.org/10.1038/s41467020-16913-2.

Correspondence and requests for materials should be addressed to C.F., J.Y. or J.M.

Peer review information Nature Communications thanks Tongmin Wang and the other, anonymous, reviewer(s) for their contribution to the peer review of this work.

Reprints and permission information is available at http://www.nature.com/reprints

Publisher's note Springer Nature remains neutral with regard to jurisdictional claims in published maps and institutional affiliations.

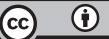

Open Access This article is licensed under a Creative Commons Attribution 4.0 International License, which permits use, sharing, adaptation, distribution and reproduction in any medium or format, as long as you give appropriate credit to the original author(s) and the source, provide a link to the Creative Commons license, and indicate if changes were made. The images or other third party material in this article are included in the article's Creative Commons license, unless indicated otherwise in a credit line to the material. If material is not included in the article's Creative Commons license and your intended use is not permitted by statutory regulation or exceeds the permitted use, you will need to obtain permission directly from the copyright holder. To view a copy of this license, visit http://creativecommons.org/ licenses/by/4.0/.

(C) The Author(s) 2020 